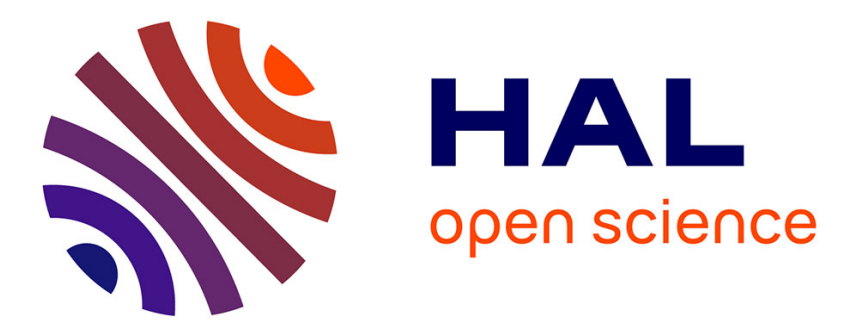

\title{
Use of Diffusion Tensor Images in Glioma Growth Modeling for Radiotherapy Target Delineation
}

Florian Dittmann, Bjoern Menze, Ender Konukoglu, Jan Unkelbach

\section{To cite this version:}

Florian Dittmann, Bjoern Menze, Ender Konukoglu, Jan Unkelbach. Use of Diffusion Tensor Images in Glioma Growth Modeling for Radiotherapy Target Delineation. Multimodal Brain Image Analysis, Sep 2013, Nagoya, Japan. pp.63-73, 10.1007/978-3-319-02126-3_7 . hal-00912667

\section{HAL Id: hal-00912667 https://hal.inria.fr/hal-00912667}

Submitted on 2 Dec 2013

HAL is a multi-disciplinary open access archive for the deposit and dissemination of scientific research documents, whether they are published or not. The documents may come from teaching and research institutions in France or abroad, or from public or private research centers.
L'archive ouverte pluridisciplinaire HAL, est destinée au dépôt et à la diffusion de documents scientifiques de niveau recherche, publiés ou non, émanant des établissements d'enseignement et de recherche français ou étrangers, des laboratoires publics ou privés. 


\title{
Use of Diffusion Tensor Images in Glioma Growth Modeling for Radiotherapy Target Delineation
}

\author{
Florian Dittmann ${ }^{1}$, Björn Menze ${ }^{2}$, Ender Konukoglu ${ }^{1}$, and Jan Unkelbach ${ }^{1}$ \\ 1 Massachusetts General Hospital, 30 Fruit Street, Boston, MA 02114, USA \\ 2 Computer Vision Laboratory, ETH Zurich, Switzerland
}

\begin{abstract}
In radiotherapy of gliomas, a precise definition of the treatment volume is problematic, because current imaging modalities reveal only the central part of the tumor with a high cellular density, but fail to detect all regions of microscopic tumor cell spread in the adjacent brain parenchyma. Mathematical models can be used to integrate known growth characteristics of gliomas into the target delineation process. In this paper, we demonstrate the use of diffusion tensor imaging (DTI) for simulating anisotropic cell migration in a glioma growth model that is based on the Fisher-Kolmogorov equation. For a clinical application of the model, it is crucial to develop a detailed understanding of its behavior, capabilities, and limitations. For that purpose, we perform a retrospective analysis of glioblastoma patients treated at our institution. We analyze the impact of diffusion anisotropy on model-derived target volumes, and interpret the results in the context of the underlying images. It was found that, depending on the location of the tumor relative to major fiber tracts, DTI can have significant influence on the shape of the radiotherapy target volume.
\end{abstract}

Keywords: glioma, tumor growth model, target delineation, diffusion tensor imaging, radiotherapy

\section{Introduction}

Gliomas, the most common primary brain tumors, grow infiltratively into the brain parenchyma. Unfortunately, conventional imaging modalities reveal only the central, highly cellular part of the tumor, whereas areas of low tumor cell density appear normal. In standard radiation treatments of high-grade gliomas, the clinical target volume (CTV) is defined by applying an isotropic $1-3 \mathrm{~cm}$ margin around the abnormality visible in MRI, to account for potential tumor infiltration in normal appearing brain [1]. However, this process hardly accounts for the growth characteristics of gliomas that are known from histopathological findings $[2,3]$ :

1. preferential spread of tumor cells along the white matter fiber tracts

2. reduced infiltration of gray matter 
3. anatomical boundaries that prevent tumor cell migration, e.g. ventricles, falx cerebri, and tentorium cerebelli

Accounting consistently for these growth patterns can result in a more personalized definition of the CTV, and therefore potentially improve the current treatment procedure. We use a phenomenological tumor growth model, which estimates a spatial tumor cell distribution by formalizing the growth characteristics. The model, which is based on the Fisher-Kolmogorov equation $[4,5]$, is personalized via the patient's MRI imaging data [6]. For radiotherapy planning, the CTV is defined as an isoline of the estimated tumor cell density obtained from the model. For a clinical application, the model is used as a tool that automatically suggests a CTV to the physician, thus making target delineation semi-automatic and more objective. To that end, it is crucial to understand the model's behavior, its capabilities, and its limitations. Previous work has conceptually introduced the Fisher-Kolmogorov model to describe glioma growth [4], including the integration of DTI [7]. However, the specific problems related to radiotherapy planning applications have not been addressed in detail.

In this paper, we evaluate the potential of the Fisher-Kolmogorov model for target delineation. By retrospective analysis of glioblastoma multiforme (GBM) patients treated at our institution, we identify the best clinical use-cases. We determine tumor locations for which the model yields target contours that are more consistent with glioma growth patterns, compared to the manually drawn contours used for treatment. The paper focuses on the use of DTI to model preferential tumor spread along white matter fiber tracts within the glioma growth model. In particular, we characterize anatomical situations in which the additional use of DTI (on top of modeling anatomical barriers and reduced gray matter infiltration) suggests different target volumes.

In section 2, we briefly summarize the underlying tumor growth model and its personalization. Section 3 discusses how DTI is incorporated to model preferential cell migration along white matter fiber tracts. We suggest a new parameterized construction of the cell diffusion tensor from the DTI data, using a single quantitatively interpretable anisotropy parameter. In section 4 , we present results for 3 patients. For one case, we present a detailed case study to explain the impact of DTI on CTV delineation. The results are interpreted in the context of the patient anatomy and the imaging data.

\section{Tumor Growth Model}

The glioma growth model formalizes the two processes that describe tumor growth: local proliferation of tumor cells and diffusion into surrounding brain tissue. It is based on the Fisher-Kolmogorov equation, a reaction-diffusion equation for the normalized tumor cell density $u=u(\boldsymbol{x}, t)$ :

$$
\frac{\partial u}{\partial t}=\nabla \cdot(D(\boldsymbol{x}) \nabla u)+\rho u(1-u)
$$


The proliferation rate $\rho$ is assumed to be spatially constant, but the $3 \times 3$ diffusion tensor $D(\boldsymbol{x})$ depends on the location $\boldsymbol{x}$. The diffusion term $\nabla \cdot(D(\boldsymbol{x}) \nabla u)$ represents the migration of tumor cells into the surrounding tissue, and the reaction term $\rho u(1-u)$ describes the cell proliferation as logistic growth.

Conversion into a static model: A direct integration of (1) is impractical, because the initial condition that corresponds to the tumor appearance in MRI at the time of treatment planning is unknown. To circumvent this problem, we use the method suggested in [5], which is based on the assumption that the visible tumor is an isoline of the cell density, and the fact that the asymptotic solution of (1) is a traveling wave. Thus, (1) can be approximated by an anisotropic Eikonal equation, which is solved by the anisotropic fast marching algorithm presented in [8]. The result is similar to the geodesic distance model in [9].

Personalization of the model: To apply the model to the patient at hand, an initial segmentation of the brain into tumor, white matter (WM), gray matter (GM), and cerebrospinal fluid (CSF) is performed, using co-registered diagnostic MR images (T1, T2, FLAIR, and T1 post contrast) as input. We utilize Expectation-Maximization-based segmentation similar to the generative tumor segmentation model of [6]. However, the initial segmentation result is enhanced as suggested in [10], in order to reliably incorporate the most prominent anatomical boundaries falx cerebri and tentorium cerebelli. The propagation of infiltration values is restricted to the WM and GM area, and CSF is used to model anatomical boundaries.

\section{Parameterization of Anisotropic Cell Diffusion}

The diffusion tensor $D(\boldsymbol{x})$ for a voxel $\boldsymbol{x}$ is constructed as

$$
D(\boldsymbol{x})= \begin{cases}D_{w} T(\boldsymbol{x}), & \boldsymbol{x} \in W M \\ D_{g} I, & \boldsymbol{x} \in G M\end{cases}
$$

where $I$ is the identity matrix, and $D_{w}$ and $D_{g}$ are diffusion rates in WM and GM, respectively. By choosing $D_{w}>D_{g}$, we model reduced infiltration of GM. In this paper, we set $D_{w} / D_{g}=100$. By constructing $T(\boldsymbol{x})$ from the water diffusion tensor $D T I(\boldsymbol{x})$ obtained from DTI, we can describe anisotropic diffusion in WM. The general idea behind the parameterization of $T(\boldsymbol{x})$ is based on the orthonormal eigenvector matrix $E(\boldsymbol{x})$ of $D T I(\boldsymbol{x})$ :

$$
T(\boldsymbol{x})=E(\boldsymbol{x})\left(\begin{array}{ccc}
\xi_{1} & 0 & 0 \\
0 & \xi_{2} & 0 \\
0 & 0 & \xi_{3}
\end{array}\right) E^{T}(\boldsymbol{x})
$$

The eigenvectors $\boldsymbol{e}_{i}$ in $E(\boldsymbol{x})$ are the eigenvectors of $D T I(\boldsymbol{x})$ with associated eigenvalues $\lambda_{i} . \xi_{1}, \xi_{2}$, and $\xi_{3}$ control the cell diffusion along these eigenvectors, where $\xi_{1}$ corresponds to the principle eigenvector $\boldsymbol{e}_{1}$. 


\section{Weighting of diffusion anisotropy:}

Setting $\xi_{i}=\lambda_{i}$ leads to $T(\boldsymbol{x})=D T I(\boldsymbol{x})$. For this naive construction, one cannot control the degree of anisotropy. Since this paper focuses on the question how diffusion anisotropy can potentially impact target volumes, a parameterization is needed that matches the following criteria: (1) one single parameter controls the amount of anisotropy, (2) the parameter is interpretable quantitatively, (3) the parameterization results in an intuitive behavior of the model.

The main idea of our parameterization is to increase the cell diffusion along the principle diffusion axis, depending on the water diffusion anisotropy and a free constant weighting parameter. To that end, $\xi_{2}$ and $\xi_{3}$ are set to $\xi_{2}=\xi_{3}=1$, whereas $\xi_{1}$ is chosen to be larger than 1 . To construct $\xi_{1}$, we first normalize $\lambda_{i}$ by using the apparent diffusion coefficient (ADC): $\bar{\lambda}_{1}=3 \lambda_{1} / \sum_{i} \lambda_{i}$. With $\bar{\lambda}_{1}$ being independent from the overall water diffusion at the location $\boldsymbol{x}$, we define $\xi_{1}$ as:

$$
\xi_{1}=1+\gamma\left(\bar{\lambda}_{1}-1\right), \gamma \geq 0
$$

The anisotropy weighting parameter $\gamma$ controls the amount of diffusion that is added to the principal axis. Furthermore, the parameterization has the following desirable properties:

1. For $\gamma=0$ the diffusion is isotropic and independent from $\operatorname{DTI}(\boldsymbol{x})$.

2. For $\gamma=1$ the diffusion along the principal axis is equal to the ADC normalized water diffusion.

3. An isotropic water diffusion results in an isotropic $T(\boldsymbol{x})$.

4. Arbitrary high anisotropy can be introduced through increasing the diffusion along the principal axis via $\gamma$.

5. Because only the cell diffusion along the principal axis is modified, it is easier to trace the influence of anisotropy on the model and on the estimated tumor cell distribution.

6. The diffusion $\xi_{1}$ along the principal axis scales linearly with $\bar{\lambda}_{1}$ and $\gamma$, which makes $\gamma$ quantitatively interpretable.

\section{Model-Based Target Delineation}

We performed a retrospective study involving six glioblastoma multiforme patients with varying tumor location treated at our institution. In this section, we report general observations from this group of patients, and illustrate relevant findings. Section 4.1 presents an in-depth analysis of one case to explain the anatomical features (major fiber tracts) that cause difference in model-derived targets when incorporated via DTI. Sections 4.2 and 4.3 show the impact of incorporating DTI data for two additional cases.

\subsection{Case I}

For case I, the tumor is located in the left parietal lobe (right side of the image) close to the corpus callosum (CC). Figure 1a displays the FLAIR image showing 
peritumoral edema, as well as the segmentation of the contrast enhancing tumor core (green), and the manually delineated CTV (red) from the actually applied treatment plan. The manually delineated CTV covers most of the left hemisphere except for the anterior part of the frontal lobe.

Figure 1c visualizes the principal eigenvector $\boldsymbol{e}_{1}$ of the diffusion tensor weighted with the normalized first eigenvalue $\bar{\lambda}_{1}$. As shown, the major fiber tracts adjacent to the tumor core are the $\mathrm{CC}$ with a dominant left-right fiber orientation (red), and the tracts with a strong superior-inferior directionality (blue), such as the corticopontine tract and corticospinal tract. The edema surrounding the tumor core leads to a low diffusion anisotropy in this area, visible as low intensity in fig. $1 \mathrm{c}$ and in the $\bar{\lambda}_{1}$ images (fig. $1 \mathrm{~d}$ to $1 \mathrm{f}$ ).

\section{Illustration of the growth model without DTI}

Figure 1b illustrates the result of the tumor growth model. It shows the isolines of the tumor cell density throughout the brain for isotropic diffusion in WM $(\gamma=0)$. The orange contour in fig. 1a represents the isoline that encompasses the same total volume as the manually drawn CTV (red). The model accounts for the falx cerebri and the ventricles as anatomical barriers, but models tumor cell migration through the CC into the contralateral hemisphere. Here, the growth model suggests an expansion of the target volume into the contralateral hemisphere. This is not accounted for in the manual delineation, which incorporates the falx cerebri as boundary but does not consistently account for the CC. In addition, the model describes the reduced infiltration of gray matter, resulting in a sharp falloff of the tumor cell density within the cortical gray matter surrounding the sulci.

\section{Influence of DTI on target volumes}

We now discuss the additional influence of DTI. For that purpose, we calculate the tumor cell densities for $\gamma=0, \gamma=5$, and $\gamma=20$. In fig. 1d to 1 f, the $\bar{\lambda}_{1}$ images are overlaid with five selected isolines. For the sake of comparison, the same color of isoline corresponds to the same size of the enclosed volume. ${ }^{3}$ In particular, the volume of the orange isolines matches the size of the manually delineated CTV. The red and green contours enclose smaller volumes, which can be used to define radiotherapy boost volumes. The contours in fig. $1 \mathrm{~d}(\gamma=0)$, serving as reference, are extracted isolines from the cell density distribution shown in fig. $1 b$.

To show the major differences between targets derived from the isotropic and anisotropic cell diffusion in more detail, figure 2 illustrates the contours, matching the size of the manual CTV, for $\gamma=0$ (yellow) and $\gamma=20$ (red) for two axial slices.

\footnotetext{
${ }^{3}$ Instead of isolines with the same infiltration value, it is indicated to compare isolines enclosing a volume of the same size, because the parameterization presented in section 3 introduces higher diffusion rates, resulting in higher infiltration values per se.
} 


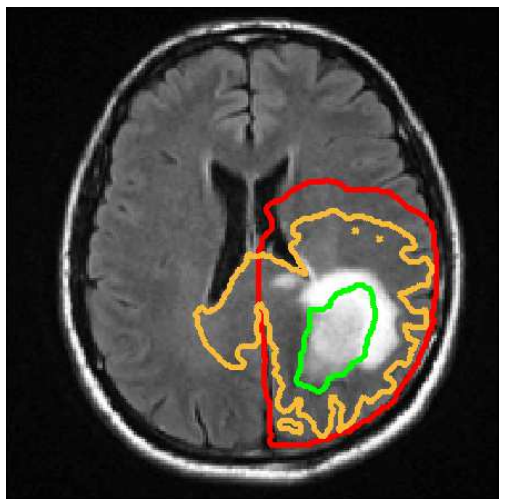

(a) FLAIR image with core (green), manual CTV (red), and modelderived CTV for $\gamma=0$ (orange)

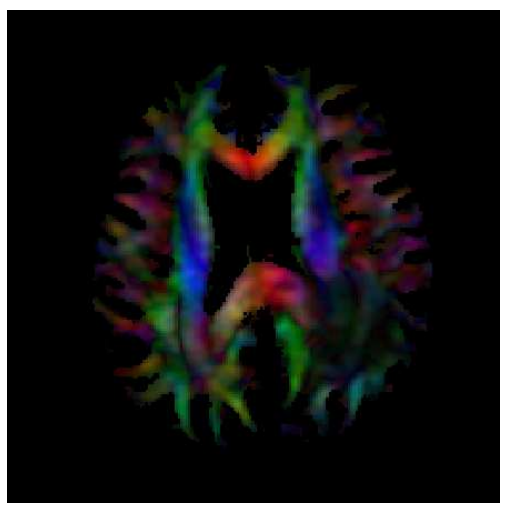

(c) Principal eigenvector $\boldsymbol{e}_{1}$ with $\bar{\lambda}_{1}$ weighting in WM

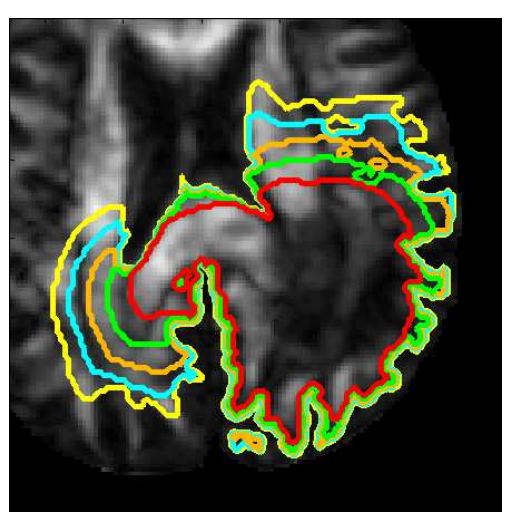

(e) $\gamma=5$ isolines on $\bar{\lambda}_{1}$

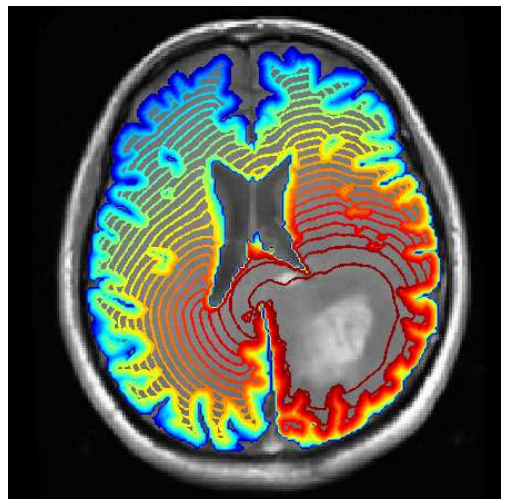

(b) Isolines of the estimated tumor cell densities $(\gamma=0)$ on $\mathrm{T} 1$ gadolinum enhanced image

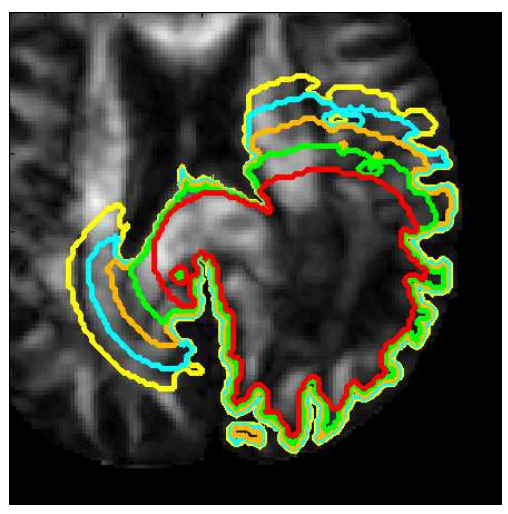

(d) $\gamma=0$ isolines on $\bar{\lambda}_{1}$

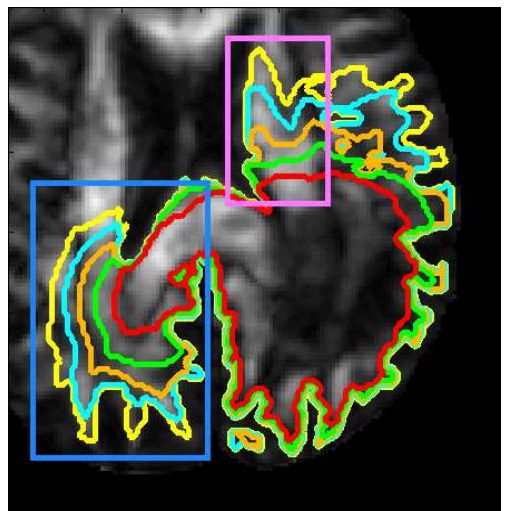

(f) $\gamma=20$ isolines on $\bar{\lambda}_{1}$

Fig. 1: Comparison of multiple isolines for $\gamma=0,5,20(\gamma=0$ corresponds to an isotropic growth model without DTI; lines of the same color correspond to the same volume; orange line corresponds to the manual CTV size) 


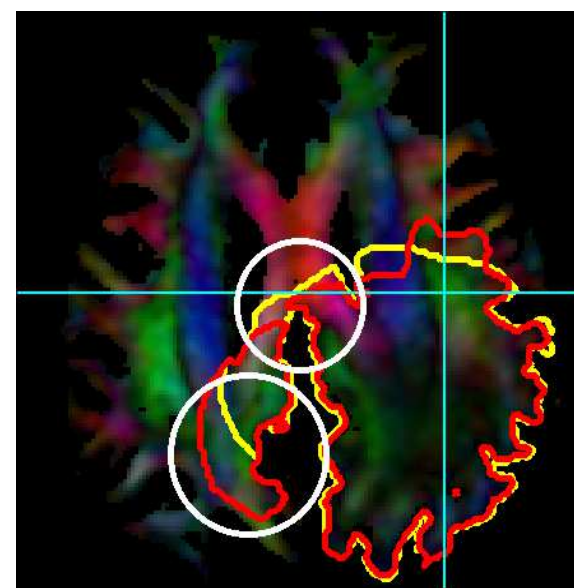

(a) Axial slice at the superior part of the corpus callosum

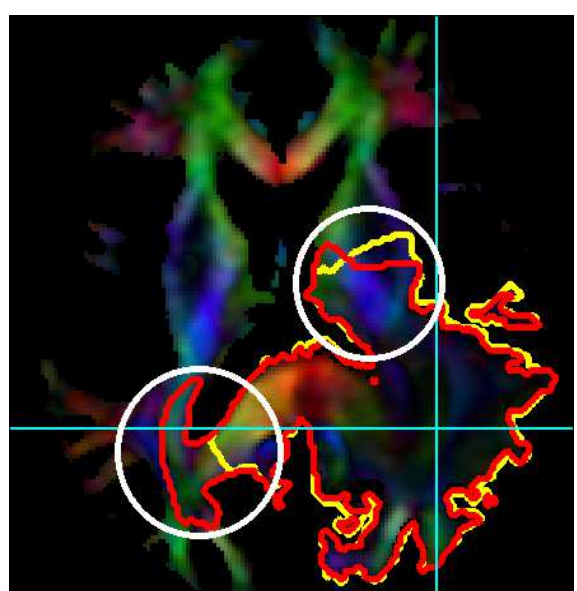

(b) Axial slice at the inferior part of the corpus callosum

Fig. 2: Comparison of the target volumes for $\gamma=0$ (yellow) and $\gamma=20$ (red) on visualization of $\boldsymbol{e}_{1}$ with $\bar{\lambda}_{1}$ weighting

Contralateral hemisphere: As shown in fig. 1, all contours extend far into the CC due to its close proximity to the tumor core. With increasing anisotropy weighting $\gamma$, the isolines extend further into the contralateral hemisphere (blue box area in fig. 1f). Here, the strong alignment of fibers in the CC results in a high diffusion anisotropy, and therefore in more infiltration of the contralateral side. Especially the blue and the yellow isolines, describing bigger volumes than the manual CTV, are further expanded. For high values of $\gamma$, they reach the smaller peripheral posterior fiber tracts of the contralateral side. The major change of the isolines is visible between the pure isotropic case $(\gamma=0)$ and the case with anisotropy weighting of $\gamma=5$. Differences between the isolines of $\gamma=5$ and $\gamma=20$ are comparatively small.

The tendency that the contralateral side is more likely to be infiltrated in the anisotropic case is also visible in the direct comparison of the contours of $\gamma=0$ (yellow) and $\gamma=20$ (red) in fig. 2a (bottom circle) and fig. 2b (left circle).

Frontal lobe: We now consider the region anterior to the tumor core (pink box area in fig. 1f). For the isotropic case $(\gamma=0$, fig. 1d), the target extension into the frontal lobe is characterized by a smooth contour (mostly defined by the geometric distance in WM from the core). In contrast, in the anisotropic case with high diffusion weighting $(\gamma=20$, fig. 1f), the isolines have a jagged shape since they incorporate the varying fiber tract orientations in this region. Especially the yellow and the blue lines tend to follow the anterior-posterior oriented fibers, i.e. the anterior part of the internal capsule, and the superior part of the fronto-occipital fascicilus (green fiber tracts in fig. 1c). This effect is 
also apparent in fig. 2. For a lower anisotropy weighting $(\gamma=5$, fig. 1e), this effect is less pronounced.

Superior part of the corpus callosum: We now compare the target volumes in the superior part of the CC (top circle in fig. 2a). In the anterior direction, the red contour $(\gamma=20)$ does not extend beyond the yellow contour $(\gamma=0)$; the yellow contour is expanded even further than red contour. This is because the dominant fiber direction (left-right) is perpendicular to the primary direction of cell migration. Since we compare contours that encompass the same volume size, and the red contour includes larger areas in the contralateral hemisphere, the target volume is to be trimmed in other regions (e.g. in the superior part of the $\mathrm{CC})$.

\subsection{Case II}

For case II, the tumor core is located in the left temporal lobe adjacient to the brain boundary. Figure 3a shows the FLAIR image in the superior part of the lesion (the green contour corresponds to the contrast enhancing core). The manually delineated CTV (red) covers large areas in the left temporal, occipital, and parietal lobes.

Figure $3 \mathrm{~b}$ compares the model derived target volumes for the isotropic case (yellow: $\gamma=0$ ) and the anisotropic case (red: $\gamma=20$ ). Both contours account for the ventricles as anatomical barrier, as well as the effect of reduced gray matter near major sulci. In this case, this becomes most apparent near the lateral sulcus.

The additional impact of DTI on the target volume is visible in the CC and the inferior fronto-occipital fasciculus. Similar to case I, the assumption of preferential spread of tumor cells along major fiber tracts modeled via DTI suggests a further expansion of the target into the CC. However, compared to case I, the effect is reduced because the tumor core is located further away from the CC.

\subsection{Case III}

For case III, the tumor core is located within the left superior frontoparietal region. The FLAIR image shows extensive peritumoral edema around the contrast enhancing core (green contour in fig. 4a). The manually delineated CTV (red) covers most of the left parietal lobe and is partially extended into the left frontal lobe.

Figure $4 \mathrm{~b}$ shows the location of major fiber tracts and compares of the modelderived target volumes (matching the size of the manual CTV) for $\gamma=0$ (yellow) and $\gamma=20$ (red). The findings for this patient are consistent with case I and II: it is illustrated that the integration of anisotropic cell diffusion leads to further extension of the isolines into the $\mathrm{CC}$ and the contralateral side. The magnitude of this effect depends on the distance of the tumor from the CC. 


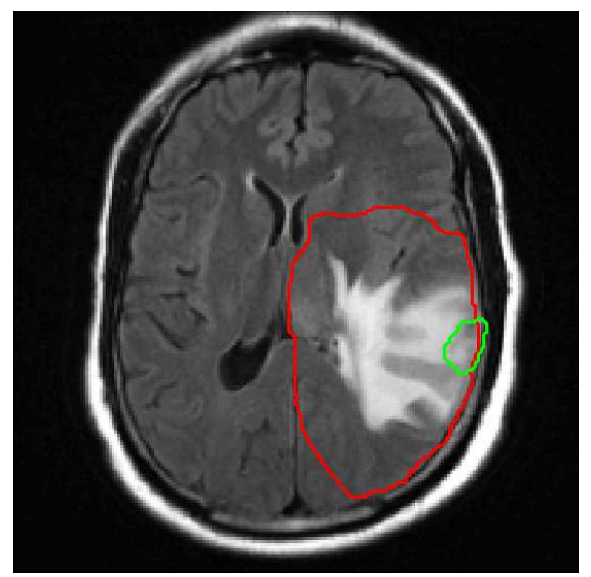

(a) FLAIR image with tumor core (green) and manual CTV (red)

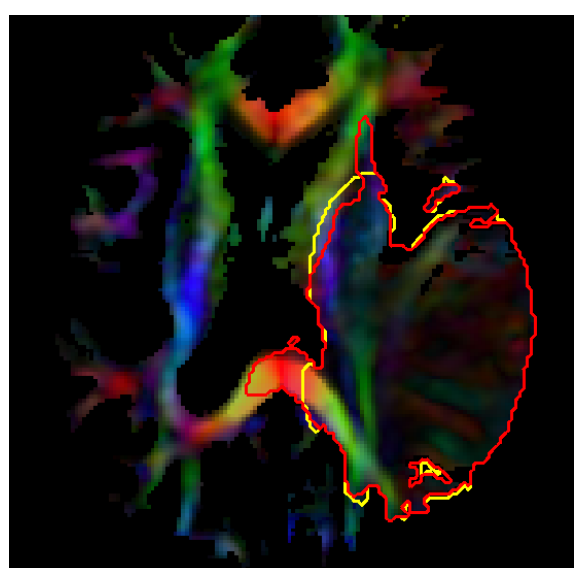

(b) Target volumes for $\gamma=0$ (yellow) and $\gamma=20$ (red) overlaid on the visualization of $\boldsymbol{e}_{1}$ with $\bar{\lambda}_{1}$ weighting

Fig. 3: Case II: Comparison of the target volumes

Case III also illustrates a limitation of the use of DTI to model preferential tumor cell migration along fiber tracts: within the edematous region the anisotropy signal obtained from DTI is reduced (visible as the hypointense region in fig. 4b). In this region, the information about the dominant fiber orientation is compromised. This reduces the differences between the isotropic and the anisotropic model.

\subsection{Application to Low-Grade Gliomas}

Model-based CTV definition for low-grade gliomas was not explicitly addressed in this paper. However, the presented methodology would be equally applicable to low-grade gliomas. In current clinical practice, the margin for the manual CTV definition in low-grade cases (1-2 cm, [11]) is smaller than in high-grade cases, resulting in a smaller volume. Therefore, for low grade-gliomas, only high anisotropy of the DTI signal in the immediate neighborhood of the MRI abnormality can have an impact on model-derived CTVs. On the other hand, accurate target delineation and optimal sparing of brain tissue is of particular interest in low-grade gliomas due to the longer life expectancy of patients.

\section{Conclusion}

The spatial growth patterns of gliomas are influenced by the preferential spread of tumor cells along the white matter fiber tracts. This can be formalized via a spatially varying, anisotropic cell diffusion tensor in a reaction-diffusion equation. In this work, we formulated a construction of the cell diffusion tensor from 


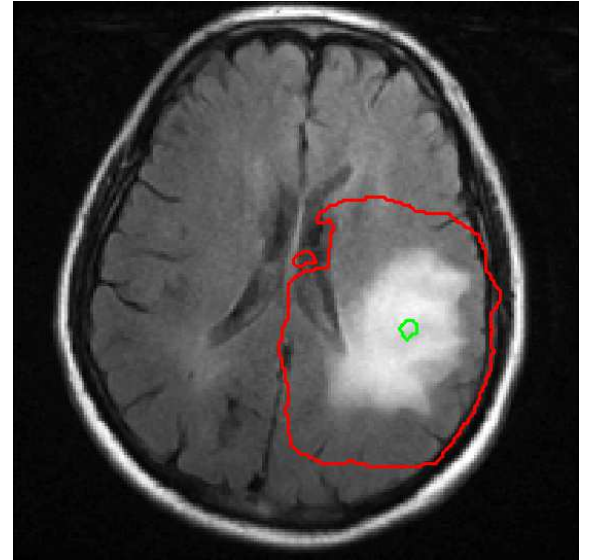

(a) FLAIR image with tumor core (green) and manual CTV (red)

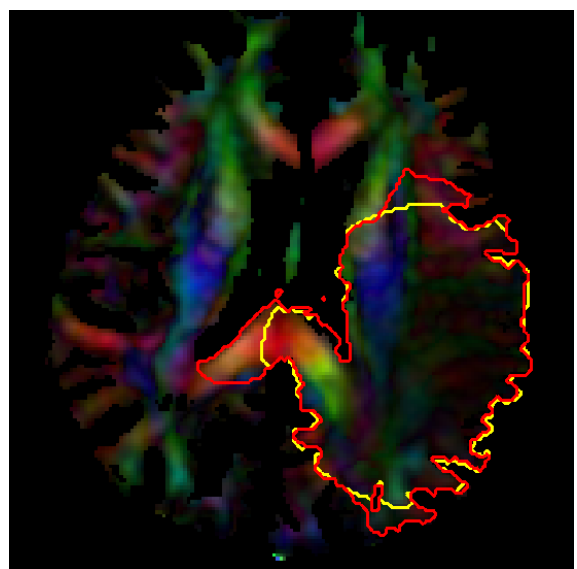

(b) Target volumes for $\gamma=0$ (yellow) and $\gamma=20$ (red) overlaid on the visualization of $\boldsymbol{e}_{1}$ with $\bar{\lambda}_{1}$ weighting

Fig. 4: Case III: Comparison of the target volumes

the DTI derived water diffusion tensor. We introduce a single anisotropy parameter $\gamma$, which controls the increased diffusion along the principle axis, i.e. the primary fiber direction.

Based on this comprehensive tensor parameterization, we investigate the influence of DTI on target delineation for radiotherapy. Six patients were analyzed. One of the main findings is that, for tumors located in proximity to the corpus callosum, an anisotropic diffusion parameterization indicates a further expansion into the contralateral side. This shows that the integration of DTI can have significant impact on the model-derived target volume for radiation therapy. Current research addresses the validation of the glioma growth model based on follow-up MR imaging and clinical outcome data.

\section{References}

1. Becker, K.P., Yu, J.: Status quo-standard-of-care medical and radiation therapy for glioblastoma. Cancer J 18(1) (2012) 12-19

2. Coons, S.: Anatomy and growth patterns of diffuse gliomas. In Berger, M., Wilson, C., eds.: The gliomas. W.B. Saunders Company, Philadelphia (1999) 210-225

3. Matsukado, Y., MacCarty, C., Kernohan, J., et al.: The growth of glioblastoma multiforme (astrocytomas, grades 3 and 4) in neurosurgical practice. Journal of neurosurgery 18 (1961) 636

4. Harpold, H.L.P., Alvord, Jr, E.C., Swanson, K.R.: The evolution of mathematical modeling of glioma proliferation and invasion. J Neuropathol Exp Neurol 66(1) (Jan 2007) 1-9

5. Konukoglu, E., Clatz, O., Bondiau, P.Y., Delingette, H., Ayache, N.: Extrapolating glioma invasion margin in brain magnetic resonance images: suggesting new irradiation margins. Med Image Anal 14(2) (Apr 2010) 111-125 
6. Menze, B., Van Leemput, K., Lashkari, D., Weber, M., Ayache, N., Golland, P.: A generative model for brain tumor segmentation in multi-modal images. MICCAI 2010 (2010) 151-159

7. Jbabdi, S., Mandonnet, E., Duffau, H., Capelle, L., Swanson, K.R., Pélégrini-Issac, M., Guillevin, R., Benali, H.: Simulation of anisotropic growth of low-grade gliomas using diffusion tensor imaging. Magn Reson Med 54(3) (Sep 2005) 616-624

8. Konukoglu, E., Sermesant, M., Clatz, O., Peyrat, J.M., Delingette, H., Ayache, N.: A recursive anisotropic fast marching approach to reaction diffusion equation: application to tumor growth modeling. Inf Process Med Imaging 20 (2007) 687-699

9. Cobzas, D., Mosayebi, P., Murtha, A., Jagersand, M.: Tumor invasion margin on a Riemannian space of brain fibers. In: LNCS 5762 Proc. MICCAI Part 2, Heidelberg, Germany, Springer (2009) pp 531-39

10. Unkelbach, J., Menze, B., Motamedi, A., Dittmann, F., Konukoglu, E., Ayache, N., Shih, H.: Glioblastoma growth modeling for radiotherapy target delineation. In: Proc MICCAI Workshop on IGRT. (2012)

11. Grier, J.T., Batchelor, T.: Low-grade gliomas in adults. The Oncologist 11(6) (2006) 681-693 